\title{
Design Thinking \& Comunicação Aumentativa e Alternativa como ferramentas para o ensino e auxílio de professores do Atendimento Educacional Especializado
}

\author{
${ }^{1,3}$ Geraldo Gomes da Cruz Júnior, ${ }^{2}$ Rafaella Leandra Souza do Nascimento, \\ ${ }^{4}$ Nadja Carneiro da Silva Gomes, ${ }^{1}$ Rinaldo José de Lima \\ ${ }^{1}$ Departamento de Estatística e Informática - Universidade Federal Rural de \\ Pernambuco (UFRPE) - Recife, PE - Brasil \\ ${ }^{2}$ Escola Politécnica de Pernambuco - Universidade de Pernambuco (UPE) - \\ Recife, PE - Brasil \\ ${ }^{3}$ Instituto SENAI de Inovação para Tecnologias da Informação e Comunicação \\ (ISI-TICs) - Universidade de Pernambuco (UPE) - Recife, PE - Brasil \\ ${ }^{4}$ Prefeitura Municipal de Igarassu - Escola Francisco Simões da Costa - \\ Igarassu, PE - Brasil \\ \{geraldoj8, rafaellalsn, rjlima01, abornadja\}@gmail.com
}

\begin{abstract}
The Specialized Educational Assistance (SEA) is a set of accessibility activities with pedagogical purposes, focused on attending students with special needs. Already the Augmentative and Alternative Communication (AAC) focuses on compensating for understanding and communication for individuals with disabilities in expression. Brazilian schools face difficulties in offering SEA with quality. Based on this, this study uses Design Thinking as an immersion approach in the problems of a rural public school to propose low-cost, efficient and sustainable solutions. By using Colorful Semantic in cards and in an application it was possible to improve indicators of learning and communication of students of the SEA.
\end{abstract}

Resumo. O Atendimento Educacional Especializado (AEE) é um conjunto de atividades de acessibilidade com fins pedagógicos, focado em atender alunos com necessidades especiais. Já a Comunicação Aumentativa e Alternativa (CAA) foca em compensar a compreensão e a comunicação para indivíduos com incapacidades na expressão. As escolas brasileiras enfrentam dificuldades em oferecer o AEE com qualidade. Com base nisto, este estudo usa o Design Thinking como abordagem de imersão na problemática de uma escola pública rural para propor soluções de baixo custo, eficientes e sustentáveis. Utilizando-se o Colorful Semantic em cartões e em um aplicativo conseguiu-se melhorar indicadores de aprendizagem e comunicação de alunos do AEE.

\section{Introdução}

No último Censo Demográfico, 45,6 milhões de pessoas declararam ter pelo menos um tipo de deficiência, seja do tipo visual, auditiva, motora ou mental/intelectual [IBGE 
VII Congresso Brasileiro de Informática na Educação (CBIE 2018)

Anais do XXIX Simpósio Brasileiro de Informática na Educação (SBIE 2018)

2010]. Esse número é equivalente a $23,9 \%$ da população do país, o que demostra a grande importância em se investir em políticas e recursos de acessibilidade para facilitar o cotidiano desse público. E uma área que carece desses investimentos é a Educação. $\mathrm{O}$ Atendimento Educacional Especializado (AEE) pode ser entendido como um conjunto de atividades e recursos de acessibilidade com fins pedagógicos, focados em atender alunos com algum tipo de necessidade especial.

O AEE tem como principal objetivo complementar a formação do estudante através de técnicas pedagógicas que ajudem esse indivíduo a ser inserido da melhor forma na sociedade [BRASIL 2009]. Porém, o que se percebe é que a construção de um caminho pedagógico para o AEE para pessoas com dificuldade de comunicação e expressão, numa perspectiva inclusiva, tem encontrado dificuldades para se efetivar, em virtude de problemas relacionados a decisões político-filosóficas, pedagógicas, metodológicas, de gestão e planejamento da escola brasileira [Damázio 2007]. O uso efetivo das tecnologias digitais pode ser um forte aliado para acessar, adaptar e construir conhecimentos no cenário AEE.

Em meio a isso, uma abordagem que vem ganhando força em todo o mundo é o uso da Comunicação Aumentativa e Alternativa (CAA). A CAA, segundo a American Speech-Language-Hearing Association [ASHA 2018], destina-se a compensar e facilitar, permanentemente ou não, prejuízos e incapacidades dos sujeitos com graves distúrbios da compreensão e da comunicação expressiva (gestual, falada e/ou escrita). É uma área da prática clínica, educacional e de pesquisa e, acima de tudo, um conjunto de procedimentos e processos que visam maximizar a comunicação, complementando ou substituindo a fala e/ou a escrita [Ciceri e Bolli Mota 2015].

Dentro da CAA, é muito difundido o uso de Símbolos de Comunicação Pictórica (SCP), que consiste em utilizar símbolos e imagens como forma de comunicação. $\mathrm{O}$ sistema SCP visa a desenhos simples e claros, de fácil reconhecimento, adequados para usuários de qualquer idade, facilmente combináveis com outras figuras e fotos para a criação de recursos de comunicação individualizados, os quais são úteis para criação de atividades educacionais [Jusoh e Majid 2017]. Um recurso complementar ao SCP é a do Colorful Semantic (CS), que é especificamente destinada a ajudar crianças a desenvolverem a sua gramática, mas enraizada ao significado semântico das palavras.

Portanto, este trabalho busca imergir e entender melhor a problemática de crianças com dificuldades de expressão no AEE, procurando formas que levem este aluno a uma aprendizagem contextualizada e significativa, valorizando seu potencial e desenvolvendo suas habilidades cognitivas, linguísticas e sócio afetivas, inserindo a tecnologia digital como potencial recurso de apoio. Para isto, esse estudo fez uso da abordagem do Design Thinking (DT), o qual coloca os usuários no centro do desenvolvimento da solução de forma colaborativa.

Através das fases do DT foi realizado um estudo de caso em uma escola pública municipal, situada na zona rural da cidade de Igarassu, Pernambuco, envolvendo alunos, responsáveis e profissionais da educação. Esse estudo buscou compreender a problemática do oferecimento do AEE nessa escola (que reflete a realidade de muitas outras escolas brasileiras), focando nos recursos disponíveis e necessidades observadas. A proposta consiste no uso do CS como uma abordagem primordial no AEE e foram desenvolvidas abordagens com cartões de papel e com o uso de um aplicativo open 
VII Congresso Brasileiro de Informática na Educação (CBIE 2018)

Anais do XXIX Simpósio Brasileiro de Informática na Educação (SBIE 2018)

source, onde os professores podem inserir os cartões de comunicação que considerem mais relevantes no dia-a-dia dos alunos, facilitando a comunicação e possibilitando a realização de atividades para o aprendizado.

\section{Trabalhos Relacionados}

Como afirma Cabral e Junior (2016), apesar dos docentes em AEE terem uma visão positiva em relação aos potenciais dos recursos tecnológicos, as práticas ainda são limitadas devido a dificuldades enfrentadas como a falta de Internet, de apoio técnico e de equipamentos, além da carência de conhecimentos sobre as tecnologias. A análise realizada por Sombrio e Rodrigues (2011), vai um pouco além, revelando que os recursos físicos, digitais, humanos e sociais não são explorados de forma adequada no AEE, o que não contribui para a construção do conhecimento.

Sobre o aspecto tecnológico e digital, trabalhos estão sendo desenvolvidos buscando analisar a aplicação das TICs (Tecnologias da Informação e Comunicação) no cenário do AEE. Em Garcia (2017) utiliza-se um estudo de caso em uma escola municipal, concluindo que a tecnologia veio somar e transformar os moldes dos atendimentos, tornando as práticas acessíveis e voltadas às necessidades de cada aluno. Ainda nesse contexto, Franco et al. (2017) apresenta uma plataforma que representa um conjunto de softwares e em nuvem para a CAA que podem ser utilizados em atividades pedagógicas como ensino de letras, números e rotinas. Segundo Moreira e Baranauskas (2016) a CAA pode ser empregada para melhorar a comunicação, além de possivelmente promover o desenvolvimento do vocabulário.

A abordagem do CS consiste em remontar sentenças as cortando em seus papéis temáticos e, em seguida, as codifica em cores. O CS vem obtendo resultados muito satisfatórios em diferentes países. Como atesta o trabalho realizado por [Hettiarachchi 2016], indicando mudanças positivas pós-intervenção nas medidas de habilidades narrativas qualitativas e quantitativas empreendidas sobre o conteúdo e estruturas sintáticas formadas pelo CS.

A literatura ratifica a utilização de conceitos de TICs, CAA e CS no contexto do AEE. Portanto, unindo as vantagens dessas técnicas e metodologias, essa pesquisa busca imergir na realidade de uma típica escola rural brasileira e propõem soluções de como esses conceitos podem ser aplicados neste contexto. São apresentadas soluções com cartões de papel e com a utilização de um aplicativo que visam a facilitação da comunicação entre professores e alunos.

\section{Metodologia}

O DT é o conjunto de métodos e processos para abordar problemas relacionados à futuras aquisições de informações, análise de conhecimento e propostas de soluções. É uma abordagem que investiga a solução de problemas de forma colaborativa, em uma perspectiva de empatia máxima com seus stakeholders, pois as pessoas são colocadas no centro do desenvolvimento da solução. O DT ajuda na imersão e no entendimento de parâmetros e padrões essenciais para criar projetos de melhor qualidade. O DT foi a abordagem básica deste projeto, pois era preciso imergir na problemática do AEE para crianças com problemas de comunicação. 
VII Congresso Brasileiro de Informática na Educação (CBIE 2018)

Anais do XXIX Simpósio Brasileiro de Informática na Educação (SBIE 2018)

Para se propor uma solução eficiente era preciso entender as principais variáveis e necessidades dos envolvidos, alunos, professores, pais, escola e governo. Alguns autores dividem o DT em 7 fases [Ambrose e Harris 2016], no entanto, normalmente considera-se 4 fases para sua implementação. Para este estudo foi utilizada uma abordagem com as fases de Imersão (entendimento), Ideação (criação), Prototipação (teste) e Desenvolvimento (aplicação), como pode ser encontrado em [SEBRAE 2017].

\subsection{Imersão}

Esta é a fase de aproximação do problema, na qual a equipe, por meio de observações e entrevistas, busca fazer o levantamento das necessidades e desafios em questão. A etapa da imersão foi dividida em duas fases: inicialmente foi realizada uma imersão preliminar e posteriormente uma em profundidade. Toda a etapa de imersão durou aproximadamente cinco meses.

$\mathrm{Na}$ imersão preliminar buscou-se o entendimento sobre a temática do AEE para crianças com dificuldade de expressão. Foi realizado um mapeamento sistemático da literatura através do Periódicos Capes e do Google Acadêmico em buscas de trabalhos e ferramentas relacionados a pesquisa. Posterior ao levantamento teórico, buscou-se identificar os principais atores envolvidos na problemática, sendo elencados: (a) alunos, (b) professores, (c) familiares, (d) escola e (e) governo.

$\mathrm{Na}$ imersão em profundidade o grupo de pesquisa do presente trabalho visitou a Escola Francisco Simões da Costa, situada na zona rural da cidade de Igarassu, Pernambuco, e analisou de perto os problemas enfrentados por alunos, professores, pais e coordenação da escola. Foram realizadas anotações diante das observações encontradas e questionários foram aplicados aos principais atores da problemática.

Todas as fases do estudo envolveram 20 alunos do AEE, 5 professoras, 10 pais e uma coordenadora da escola. A faixa etária dos alunos envolvidos consistiu entre 4 e 12 anos, onde encontra-se indivíduos com incapacidade total ou parcial de falar (mudez), Transtornos do Espectro Autista (TEA) e Síndrome de Down.

Durante a fase de imersão foi elaborado um teste de conhecimentos básicos para crianças em parceria com as professoras, o qual foi aplicado aos alunos e coletados os resultados. Também foram observados aspectos relativos à infraestrutura da escola, recursos disponíveis e programas do governo de incentivo ao AEE. A existência do Programa Dinheiro Direto na Escola (PDDE) é uma importante fonte de recursos para a manutenção dos recursos de acessibilidade existentes na escola em estudo.

Ao final desta fase, foi constatado que a situação encontrada na escola foi semelhante ao reportado pela literatura. Em termos de infraestrutura e recursos para garantir a qualidade do AEE ainda falta muito investimento. Das 5 professoras entrevistadas, apenas uma é especializada para o AEE, no entanto, a escola e professores tentam oferecer o melhor ensino possível com os recursos que têm disponíveis.

\subsection{Ideação}

Na ideação, as ideias são apresentadas sem julgamento, propondo soluções para o problema. A fase de Ideação durou aproximadamente um mês e durante esta etapa, foi realizada a sondagem dos dados e resultados colhidos e observados durante a fase de 
VII Congresso Brasileiro de Informática na Educação (CBIE 2018)

Anais do XXIX Simpósio Brasileiro de Informática na Educação (SBIE 2018)

Imersão. Esta fase envolveu a equipe do projeto, professoras e coordenadora e buscando extrair insights dos dados coletados. O grande foco desta fase foi reunir a equipe do projeto com especialistas para analisar o problema, a opinião dos envolvidos e propor soluções que se encaixassem com a realidade enfrentada pela escola. Infraestrutura, quantidade de professores, necessidades dos alunos e recursos financeiros e de acessibilidade disponíveis foram pontos chaves para se propor soluções.

A realização de brainstormings e sessões de co-criação com os envolvidos foram de grande importância, pois possibilitou desenhar possíveis soluções que atendessem ao problema. Promovendo o debate de ideias, chegou na proposta de utilização de abordagens com SCP e o CS, por serem metodologias reconhecidas internacionalmente como ferramentas de melhora na comunicação e aprendizado. Essas ferramentas podem ser aplicadas com um investimento baixo, pois pode-se imprimir os cartões de comunicação em papel, por exemplo. A literatura também é vasta em considerar uma melhora efetiva no CS quando aplicado em conjunto com abordagens tecnológicas, a maioria destas envolvendo tablets. Isso acontece por esses recursos tecnológicos estimularem os sentidos das crianças, possibilitando uma atenção maior por parte destas.

O uso do CS em cartas de papel é de fácil acesso para a escola, pois os professores podem confeccionar os cartões e imprimi-los. Porém, a utilização de uma aplicação via tablet é muito relevante para o aprendizado dos alunos, a aplicação em relação aos cartões possui recursos audiovisuais como mais luz, sons e ações táteis que são importantes para o entretenimento da criança enquanto aprende e se comunica. No entanto, houve um levantamento das principais necessidades das crianças reportadas pelos professores possibilitando que, tanto a abordagem com cartões quanto a abordagem com o aplicativo, fossem adaptadas aos inputs dos professores e as necessidades de cada criança em específico.

\subsection{Prototipação}

A prototipação é a fase de validação das ideias geradas e verificar quais delas se encaixam ao projeto. Neste trabalho, a fase de prototipação perdurou dois meses e nesse período foram desenvolvidas as duas soluções levantadas durante a fase de Ideação: (a) uma solução de CS com cartões impressos em papel; (b) e uma solução de CS através de um aplicativo open source que pudesse ser executado em tablets de baixo custo.

Para ambas as soluções foram considerados aspectos apontados como essenciais pela literatura e por especialistas, mas também foram adicionados conceitos colhidos pelos pais e professores, como sendo os principais recursos de comunicação que os estudantes iriam precisar no dia-a-dia. Foram definidas as principais cartas de cada fase do CS (QUEM - Laranja, O QUE FAZ - Amarelo, O QUE - Verde e ONDE - Azul), como também suas imagens de representação e nomes. Também foi considerado que houvesse uma forma simples dos professores conseguirem adicionar novos cartões e adaptá-los para cada aluno.

O aplicativo foi desenvolvido com tecnologias web tais como HTML, CSS e Javascript, o que possibilita a execução em diferentes dispositivos e sistemas operacionais. Além disso, sua interface responsiva garante que ele possa ser executado com qualidade em um computador, tablet ou celular. Ao fim do desenvolvimento dos 
VII Congresso Brasileiro de Informática na Educação (CBIE 2018)

Anais do XXIX Simpósio Brasileiro de Informática na Educação (SBIE 2018)

protótipos, ambos foram levados a escola para serem testados, validados e ajustados diante dos reports dos professores e alunos.

\subsection{Desenvolvimento}

Após as validações obtidas na fase prototipação, inicia-se a fase de desenvolvimento, onde o foco é a aplicação da solução proposta. Na realização deste trabalho, a fase de desenvolvimento durou aproximadamente quatro meses e nela foi mensurada e observada a aplicação das ferramentas desenvolvidas na escola, coletando feedbacks dos alunos, professores, pais e coordenação para a melhoria das ferramentas e análises do estudo. Essa fase focou na implantação e garantia de sustentabilidade da solução na escola. Também foi realizado um questionário com os alunos, elaborado em parceria com os professores e com nível equivalente ao aplicado no início do experimento. Esta avaliação foi um dos critérios utilizados para mensurar a qualidade da solução proposta.

Por fim, a equipe analisou os resultados das entrevistas, feedbacks e avaliações finais em conjunto com o corpo docente, pais e coordenadora. Ponderou-se sobre os aspectos de qualidade alcançados com a aplicação das ferramentas na escola e concluise que a solução proposta alcançou resultados significativos para a melhora na aprendizagem de crianças do AEE. Os resultados serão descritos no capítulo seguinte.

\section{Resultados e Discussões}

Este estudo possibilitou a coleta de resultados interessantes, oriundos de cada uma das fases da abordagem do DT. Porém, aqui serão ilustrados e discutidos os resultados considerados como mais relevantes pela equipe do projeto.

Durante a etapa de imersão, a equipe do projeto através das entrevistas e observações buscou encontrar respostas que atendessem aos seguintes filtros de pesquisa, divididos em três grupos de análises, como descritos na Tabela 1.

Tabela 1. Categorias analisadas para a realização da pesquisa.

\begin{tabular}{c|l}
\hline \multicolumn{1}{c}{ Grupo } & \multicolumn{1}{c}{ Descrição } \\
\hline $\begin{array}{l}\text { Em relação } \\
\text { aos alunos }\end{array}$ & $\begin{array}{l}\text { Foi observado quais os materiais e equipamentos disponíveis na escola para o AEE. Quais } \\
\text { as atividades realizadas neste ambiente, bem como quais elementos são utilizados para } \\
\text { estas atividades }\end{array}$ \\
\hline $\begin{array}{l}\text { Em relação } \\
\text { aos pais e } \\
\text { professores }\end{array}$ & $\begin{array}{l}\text { Foi observado quais os materiais e equipamentos disponíveis na escola para o AEE. Quais } \\
\text { as atividades realizadas neste ambiente, bem como quais elementos são utilizados para } \\
\text { estas atividades }\end{array}$ \\
\hline $\begin{array}{l}\text { Em relação } \\
\text { à escola }\end{array}$ & $\begin{array}{l}\text { Foi observado quais os materiais e equipamentos disponíveis na escola para o AEE. Quais } \\
\text { as atividades realizadas neste ambiente, bem como quais elementos são utilizados para } \\
\text { estas atividades }\end{array}$ \\
\hline
\end{tabular}

Pode-se perceber que são poucos os recursos disponíveis para o AEE e que a escola tenta fazer o melhor uso possível das verbas recebidas pelo governo, porém é perceptível o quanto a dificuldade de expressão atrapalha os alunos tanto no seu aprendizado, como no seu dia a dia. Este estudo buscou concretizar soluções dentro da realidade de recursos da escola, com foco em inserir este aluno de uma melhor forma na sociedade e em um ambiente de aprendizado. Buscou-se possibilitar maior interação dos alunos do AEE com os demais colegas, como as atividades de brincadeira poderiam ser 
VII Congresso Brasileiro de Informática na Educação (CBIE 2018)

Anais do XXIX Simpósio Brasileiro de Informática na Educação (SBIE 2018)

adaptadas para que todos possam participar e quais instrumentos possibilitariam esta participação e colaboração de todos.

Ao final das entrevistas e observações, pode-se levantar as necessidades de comunicação mais usuais dos estudantes em um ambiente escolar. Desta forma pode-se gerar um modelo-base de vocabulário para todos os alunos, no qual os professores podem adicionar ou remover alguma palavra realizando uma análise específica e pontual por aluno. A Figura 2 exemplifica a ilustração base por trás de algumas das cartas de comunicação. O vocabulário base levantado, seguindo o modelo do CS, consiste em:

-Quem (cor laranja): Eu, você, nós, a professora, meu pai, minha mãe, minha família, a tia da cantina, a coordenadora, ele, ela, amigo, isto e aquilo;

-O que faz (cor amarela): Beber, comer, deitar, escrever, sentar, ler, desenhar, contar, cantar, dançar, lavar, colar, cortar, pintar, assistir, limpar, rir, chorar, correr, brincar, dar, sair, passear, pegar, ajudar, procurar, gostar, pensar, querer, escutar, responder, falar e ir;

-O que (cor verde): Mesa, cadeira, chão, papel, livro, caderno, cama, suco, lanche, árvore, brinquedo, lápis, água, pão, fruta, biscoito, história, atividade, prato, copo, bolsa, alfabeto, números, tablet, celular, estojo, tesoura, cola, borracha, remédio, imagem, foto e caneta;

- Onde (cor azul): Na sala, no parque, na cozinha, na escola, no banheiro, em casa, no quarto, na escada, no pátio, na saída, na igreja, no hospital, na loja e na cidade.
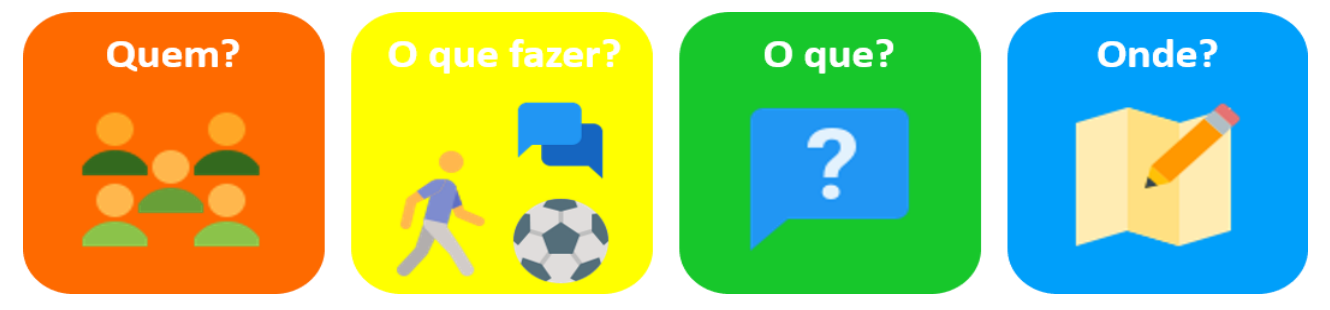

Figura 2. Exemplo da base das ilustrações dos cartões de comunicação.

Com base nesses vocabulários os alunos podem escolher entre as cartas e montarem frases para se expressarem de uma forma mais fácil e assertiva com os colegas, pais e professores. Um simples desejo de desenhar pode ser expresso pelos cartões montando uma frase de "Eu quero desenhar no papel", facilitando os desejos do aluno com as atividades propostas pelo professor do AEE.

Com os feedbacks dos envolvidos, observou-se a necessidade da criação de um novo grupo de palavras, onde nesse grupo são encaixadas ações com teor de urgência, ou de interpretação direta frente a exibição de só um cartão. Essa nova categoria, não prevista no CS, foi destacada na cor vermelha e é utilizada pelos alunos para interagirem a respostas e a necessidades mais urgentes. Esse vocabulário foi dividido em dois grupos, o primeiro para respostas de perguntas dos professores e o segundo para necessidades dos alunos. No primeiro grupo encontra-se, por exemplo: as letras do alfabeto, os números, animais, cores e objetos (que são úteis para responder perguntas). No segundo grupo pode-se descrever: Por favor repita o que disse; Eu não entendi; Eu entendi; Que horas são?; Onde fica o...?; Como fazer...?; Estou com dor; Estou bem; Estou triste, dentre outras opções. 
VII Congresso Brasileiro de Informática na Educação (CBIE 2018)

Anais do XXIX Simpósio Brasileiro de Informática na Educação (SBIE 2018)

Foi observado que a literatura indica constantemente que o uso de tablets com aplicativos voltados para o público com necessidades especiais traz um ganho positivo em relação ao aprendizado e interesse deste público pelo ato de aprender pela ferramenta [Carniel 2017]. O uso de aplicativos estimula habilidades visuais, auditivas e perceptivas dos estudantes, possibilitando um maior interesse deste indivíduo.

Pode-se identificar diversas ferramentas na literatura e no mercado que são preparadas para lidar com o CAA, elas não foram utilizadas nesse estudo devido a dois grandes fatores observados na etapa de imersão: I) a escola é situada em uma zona rural, o vocabulário e nível de experiência com interfaces de dispositivos eletrônicos observados no ambiente foi diferente do observado em outros experimentos da literatura; II) as aplicações que melhor se encaixaram para a problemática, devido a possibilidade de se customizar os textos por alunos, não são gratuitas, o que gera um entrave na compra de licenças de uso por parte da escola. Devido a esses dois pontos optou-se por o desenvolvimento de uma solução adaptada a realidade da escola.

Foi desenvolvido um aplicativo que apresenta e possibilita as mesmas ações da ferramenta anterior (construída em cartões de papel). O professor pode adicionar novos cartões de comunicação, em grupos de fala diferentes e customizar cartões por alunos. $\mathrm{O}$ aplicativo é completamente gratuito e customizável, para a realização do experimento a equipe utilizou 5 tablets de valor estimado em $\mathrm{R} \$ 150,00$. Os dispositivos utilizados no experimento foram cedidos pela equipe do projeto, mas se pensou em hardwares que estivessem acessíveis para a aquisição da escola em breve, através de verbas do governo.

Durante a fase de desenvolvimento comparou-se os resultados das entrevistas realizadas no início e próximo ao término do trabalho. A maioria das análises realizadas foram de teor qualitativo e pode-se perceber que $100 \%$ dos entrevistados, entre pais, professores e coordenadora, apontaram que o uso das ferramentas propostas proporcionou uma melhora significativa na forma como os alunos estão se expressando no seu cotidiano.

Quando se leva em consideração a atividade aplicada no início e no término do projeto, também percebe-se uma melhora no aprendizado dos alunos e em como eles conseguiram comunicar as suas respostas. No primeiro teste apenas 3 dos 20 alunos obtiveram resultado satisfatório ao teste, já após a aplicação das ferramentas, percebeuse que ficou mais claro para os estudantes como responder as questões e como lidar com o problema para o resolver. Dos 20 alunos testados 14 tiveram resultado satisfatório.

Outra análise realizada foi em relação a qual ferramenta proporcionou o melhor aprendizado, os cartões em papel, ou o aplicativo. Apenas 5 tablets estavam disponíveis para o experimento, então estes eram sorteados entre os alunos durante as atividades do AEE. Pode-se observar que sempre os estudantes com os tablets eram os mais animados em aprender e em participar das atividades. Os recursos oferecidos pela aplicação aparentam ser poderosos para a captação da atenção das crianças e para a facilitação do seu aprendizado, pois obtiveram resultados melhores nas atividades quando estavam com o tablet, após passarem por uma curva de aprendizagem da utilização do aplicativo. 
VII Congresso Brasileiro de Informática na Educação (CBIE 2018)

Anais do XXIX Simpósio Brasileiro de Informática na Educação (SBIE 2018)

\section{Conclusões}

Com a realização deste trabalho foi possível compreender melhor aspectos do ensino AEE, através do estudo de caso desenvolvido. O processo do Design Thinking aplicado tornou possível a imersão na problemática deste cenário, na qual a realização das quatro fases (Imersão, Ideação, Prototipação e Desenvolvimento) possibilitou a criação de uma aplicação que se adequasse as necessidades do público-alvo abrangido (alunos, educadores, pais e escola).

A aplicação desenvolvida foi apoiada por abordagens e recursos muito difundidos e fundamentados no cenário AEE, como a Comunicação Aumentativa e Alternativa (CAA), Símbolos de Comunicação Pictória (SCP) e o Colorful Semantic (CS). Tais conceitos, de forma geral, buscam facilitar a compreensão e a comunicação de indivíduos com dificuldade de expressão, seja ela gestual, falada ou escrita. Outros estudos já demostraram resultados satisfatórios com a aplicação destas técnicas, a contribuição deste trabalho parte da aplicação de uma metodologia embasada no Design Thinking para a construção de uma solução que atende as necessidades de turmas do AEE de uma típica escola rural brasileira. Foram levantados e considerados diferentes aspectos de recursos existentes na escola para se propor soluções de baixo custo, eficientes e sustentáveis.

Como resultados, após a validação na fase de prototipação, o aplicativo idealizado sofreu os ajustes necessários de acordo com os feedbacks dos alunos, professores e pais. Com isso, foi desenvolvida uma aplicação final mais adequada aos usuários. Os resultados de todas as fases deste trabalho foram muito positivos, pois houve a colaboração e comprometimento por parte das pessoas envolvidas na disponibilidade das informações requeridas, além do apoio conceitual referenciado.

Também pode ser observado uma melhora no aprendizado dos alunos e na forma de comunicação destes, principalmente utilizando a aplicação desenvolvida por meio de tablets. Estes resultados corroboram com a literatura, a qual utiliza os conceitos de TICs, CAA e CS no contexto do AEE como forma de promover melhorias no aprendizado e na comunicação dos alunos deste cenário.

Como trabalhos futuros pretende-se aperfeiçoar o aplicativo, inclusive através de novas funcionalidades como módulos de atividades, onde os alunos possam resolver questões e aprender novos conceitos. De forma geral, pretende-se manter proximidade com o público-alvo em questão, em um processo contínuo de validação das melhorias, feedbaks e implementações. Outro ponto importante consiste em aplicar a ferramenta proposta em outras escolas que possuam o AEE.

\section{Referências}

Ambrose, G., Harris, P. (2016) Design Thinking: Coleção Design Básico. Bookman Editora.

ASHA (2018) American Speech-Language-Hearing Association. Disponível em: $<$ http://www.asha.org/>.

BRASIL (2009) Resolução CNE/CEB n. ${ }^{\text {4, de }} 2$ de outubro de 2009. Institui Diretrizes Operacionais para o Atendimento Educacional Especializado na Educação Básica, modalidade Educação Especial. Diário Oficial da União, 5 de outubro de 2009. 
VII Congresso Brasileiro de Informática na Educação (CBIE 2018)

Anais do XXIX Simpósio Brasileiro de Informática na Educação (SBIE 2018)

Cabral, M. S. N. e Junior, J. B. B. (2016) Práticas de Ensino e Uso das Tecnologias no Atendimento Educacional Especializado: enfoque nas salas de recursos multifuncionais. Revista Novas Tecnologias na Educação, v. 14, n.1.

Carniel, A., Berkenbrock, C. D. M., \& da Silva Hounsell, M. (2017). Um mapeamento sistemático sobre o uso da comunicação aumentativa alternativa apoiada por recursos tecnológicos. Revista Brasileira de Computação Aplicada, 9(2), 84-98.

Ciceri Cesa, C. e Bolli Mota, H. (2015) Comunicação aumentativa e alternativa: panorama dos periódicos brasileiros. Revista CEFAC, v. 17, n. 1.

Damázio, M. F. M. (2007) Atendimento educacional especializado. Paraná: Cromos.

Garcia, T.G.R. (2017) O uso das TICs na prática do atendimento educacional especializado (AEE). Monografia do Curso de Especialização Tecnologias da Informação e Comunicação Aplicadas à Educação. Universidade Federal de Santa Maria, Rio Grande do Sul.

Franco, N., Lima, T., Lima, A., Silva, E., Lima, R., Cavalcante, T., \& Fidalgo, R. (2017) aBoard: Uma Plataforma para Educação Inclusiva a partir de Comunicação Aumentativa e/ou Alternativa. In Simpósio Brasileiro de Informática na EducaçãoSBIE, Vol. 28, No. 1, p. 977.

Hettiarachchi, S. (2016) The effectiveness of Colourful Semantics on narrative skills in children with intellectual disabilities in Sri Lanka. Journal of Intellectual Disabilities, v. 20, n. 1, p. 18-33.

IBGE (2010) Instituto Brasileiro de Geografia e Estatística: Censo Demográfico 2010. Rio de Janeiro: IBGE. Disponível em: <https://censo2010.ibge.gov.br/>.

Jusoh, W. and Majid, R. A. (2017) Using Picture Exchange Communication System to Improve Speech Utterance Among Children With Autism. Journal of ICSAR, v. 1, n. 1, p. 46-49.

Moreira, E., e Baranauskas, M. C. (2016) Investigando processos de comunicação alternativa via tecnologia tangível: um estudo exploratório. In Simpósio Brasileiro de Informática na Educação-SBIE, Vol. 27, No. 1, p. 856.

SEBRAE (2017) Serviço Brasileiro de Apoio às Micro e Pequenas Empresas: Entenda o Design Thinking. Disponível em: <http://www.sebrae.com.br/sites/PortalSebrae/artigos/entenda-o-designthinking,369d9cb730905410VgnVCM1000003b74010aRCRD>. Acesso em: 15 mai. 2018 .

Sombrio, C. M. e Rodrigues, A. P. (2011) O uso das TICs nas salas de recursos TGD. Revista Novas Tecnologias na Educação, v. 9, n. 1. 